Check for updates

Cite this: RSC Adv., 2019, 9, 27961

Received 24th May 2019

Accepted 29th August 2019

DOI: $10.1039 /$ c9ra03914g

rsc.li/rsc-advances

\section{Fabrication of detonation nanodiamondasodium alginate hydrogel beads and their performance in sunlight-triggered water release $\uparrow$}

\author{
Dan Zheng, ${ }^{\text {ab }}$ Bo Bai, (iD *abcd Xiaohui $\mathrm{Xu}^{\text {ab }}$ Yunhua $\mathrm{He}^{\text {ab }}$ Shan $\mathrm{Li}^{\text {ab }} \mathrm{Na} \mathrm{Hu}{ }^{\mathrm{cd}}$ \\ and Honglun Wang ${ }^{\text {cd }}$
}

\begin{abstract}
Agricultural water use accounts for around $70 \%$ of total water use in the world. Enhancing agricultural water use efficiency is a key way to cope with water shortage. Here, sunlight-responsive hydrogel beads consisting of sodium alginate (SA) matrix and detonation nanodiamond (DND) were fabricated by an ion gelation technique, which has potential applications in controlled water release. The interaction between the DND and SA matrix was investigated by Fourier transform infrared (FTIR) spectra and X-ray diffraction (XRD). UV-vis diffuse reflectance spectra verified DND can absorb solar energy in the UV, visible and even near-infrared regions. DND dispersed in the hydrogel matrix can absorb sunlight and generate heat, increasing the temperature of the matrix and resulting in slow release of water from the elastic beads. In addition, the effects of DND content and $\mathrm{pH}$ were systematically studied to evaluate their water adsorption properties. The swelling kinetics of DND@SA hydrogel beads in distilled water could be fitted well with a pseudo-second-order kinetic model. Six consecutive cycles of water release-reswelling indicated that their easy regeneration and reusability. The novel and eco-friendly hydrogel beads should be applicable to on-demand, sequential, and long-term release of water via light exposure.
\end{abstract}

\section{Introduction}

Water is one of the fundamental components of life. Especially in arid areas, water is recognized as the most effective element for improving agricultural production. Under the normal conditions of agroecosystems, water shortage would limit crop growth, while excessive use of water would increase production costs and even pollute the environment due to enhanced fertilizer leaching. ${ }^{1-3}$ Hereby, the improvement of agricultural water use efficiency is becoming a key approach to reduce irrigation water consumption, mitigate water shortage and reduce environmental problems. In order to achieve these goals in the field of agriculture, hydrogels are deemed to be a potential candidate because of their water-adsorbing capacity and slowrelease function by virtue of their elastic networks. ${ }^{4-6}$ In particular, stimulus-responsive hydrogels that possess

${ }^{a}$ Key Laboratory of Subsurface Hydrology and Ecological Effects in Arid Region of the Ministry of Education, Chang'an University, No. 126 Yanta Road, Xi'an 710054, Shanxi, China.E-mail: baibochina@163.com

${ }^{b}$ College of Environmental Science and Engineering, Chang'an University, Xi'an, 710054, P. R. China

${ }^{c}$ Key Laboratory of Tibetan Medicine Research, Northwest Institute of Plateau Biology, Chinese Academy of Sciences, Xining, 810008, China

${ }^{d}$ Qinghai Provincial Key Laboratory of Tibetan Medicine Research, Xining, 810001, P. R. China

† Electronic supplementary information (ESI) available. See DOI: 10.1039/c9ra03914g a controlled release behavior in response to environmental changes, such as temperature, ${ }^{7,8} \mathrm{pH},{ }^{\mathbf{9}, 10}$ and salt, ${ }^{11}$ etc., have attracted research interest due to their extended adaptability. Typically, they have been widely applied in the agriculture sector for controlling water release, like flower planting, greenhouse cultivation, and seed propagation, etc. Compared with these great advances, it should be noted that manipulating the extrinsic parameters of whole farmland accurately to sustain a controlled water release in a cost-effective and convenient way is still a challenging job.

The light-responsive hydrogel consisting of photothermal conversion materials with polymer matrix ${ }^{\mathbf{1 2 - 1 4}}$ is an ideal controlled water delivery platform, due to its safety and abundance during daily activity and potential for controlled release. In practical applications, the rate of water release of the lightresponsive hydrogel can be further tuned facilely and remotely through several light parameters, such as power density, wavelength, and exposure time, etc. The critically used photothermal conversion materials include carbon nanotubes, ${ }^{15,16}$ graphene oxide (GO), ${ }^{17,18}$ or metallic nanoparticles such as iron oxide ${ }^{19,20}$ or gold..$^{21-23}$ In contrast, most of them are subject to serious limitations such as a relatively low photothermal conversion efficiency, limited absorption range, which remains a practice challenge for agriculture applications. Alternatively, detonation nanodiamond (DND) formed by an $\mathrm{sp}^{3}$-carbon diamond core and a reconstructed $\mathrm{sp}^{2}$-carbon surface layer have recently received great attention on account 
of their inherent features, including high biocompatibility, large surface area, high thermal conductivity, spherical morphology, and surface functionality. ${ }^{24-26}$ In additionally, DND is an excellent photothermal material with an absorption range spanning across the UV, visible and even near-infrared regions. Owing to these excellent properties of strong absorption and favorable light and thermal stability, DND has been widely utilized for photothermal therapy by generating hyperthermia under illumination to kill tumor cells. ${ }^{27,28}$ Moreover, benefiting from their plentiful chemically active carboxyl-, hydroxyl-, carbonyl-, nitrogen-, and sulfur-groups around the outer surface of DND substances, ${ }^{29}$ DND also has acted as a functional filler in the production of composites. ${ }^{30-32}$ In a few words, the mutually beneficial characteristics suggest that DND can be easily encapsulated into hydrogels, and thereby impart excellent photothermal performance to a hydrogel, consequently realizing sunlight-controlled water releaser property. However, to date, the synthesis of hybrid photothermal conversion hydrogel materials using the DND is rarely reported.

Sodium alginate (SA) is a polyanionic linear copolymer of 1,4-linked- $\alpha$-L-guluronic acid and $\beta$-D-mannuronic acid residues found in brown seaweed. ${ }^{33}$ By virtue of its biocompatibility, biodegradability, hydrophilicity, and its remarkable gel properties in the presence of divalent cations, the sodium alginate has been regarded as a brilliant polysaccharide for water management. ${ }^{34-36}$ Based on these considerations, herein we fabricated novel detonation nanodiamond@sodium alginate hydrogel beads (DND@SA), which can be used as a sunlightcontrolled release targeted system for water. The core of our innovation is that the sunlight-responsive DND particles were encapsulated by a SA polymer matrix, and the as-obtained samples were used as both the medium of the water and the photothermally sensitive gatekeeper. The interaction between SA and DND and the structural, photothermal properties of the composites were investigated. The experimental results have verified that the as-obtained DND@SA hydrogel beads have excellent water adsorption under slightly alkaline conditions. The mechanisms lie is that DND dispersed within the hydrogel substrate can efficiently convert photo energy into thermal energy, and consequently heat up the hydrogel matrix, resulting in water release from hydrogel beads via sunlight irradiation. Such phenomena have given us a belief that the hydrogel beads are very promising for use as water-controlled release agents to ameliorate the utilization efficiency of water.

\section{Experimental section}

\subsection{Materials}

Sodium alginate and sodium hydroxide were provided by Tianjin Chemical Reagent Factory (Tianjin, China). Nanodiamond with individual sizes of 5-15 $\mathrm{nm}$ synthesized by detonation techniques were supplied by Yilin, Inc. (Shaanxi, China). Calcium chloride and hydrochloride acid were furnished by Xi'an Chemical Reagent Factory (Shaanxi, China). All agents were of analytical grade and used without further purification.

\subsection{Preparation of DND@SA hydrogel beads}

The composite solutions were prepared by simple mixing of aqueous DND dispersions with the SA, thus entrapping the DND within the hydrogel matrix. Detonation nanodiamond (0.2 g) was dispersed in $50 \mathrm{~mL}$ of distilled water, kept in an ultrasonic bath for $30 \mathrm{~min}$ to uniformly disperse it. DND dispersions were used to configure solutions with different DND content. Sodium alginate $(0.25 \mathrm{~g})$ was placed in a beaker and then a certain amount of detonation nanodiamond dispersion solution and distilled water shown in Table 1 were added with constant stirring at room temperature to form $10 \mathrm{~mL}$ mixed solution. The obtained mixture was ultrasonicated for $30 \mathrm{~min}$ and stirred for $2 \mathrm{~h}$ at room temperature to obtain a homogeneous mixture. Then, after stewing for $1 \mathrm{~h}$, the obtained products were added dropwise into $3 \%(\mathrm{w} / \mathrm{v}) \mathrm{CaCl}_{2}$ aqueous solution using a $0.8 \mathrm{~mm}$ gauge syringe without the needle under continuous magnetic stirring $(100 \mathrm{rpm})$. Mixture droplets were precipitated to form spherical gel beads in $\mathrm{CaCl}_{2}$ solution due to the ionic crosslinking between carboxyl groups of SA and $\mathrm{Ca}$ (II) ions. These hydrogel beads were allowed to harden for $12 \mathrm{~h}$ in the gelling solution, filtered and washed thoroughly with distilled water to remove the residual $\mathrm{CaCl}_{2}$ solution on the surface, and subsequently, detonation nanodiamond@sodium alginate (DND@SA) beads were obtained. Blank SA beads were obtained by a similar preparation process without adding DND.

\subsection{Measurement of photothermal performance}

2.3.1. Photothermal performance of detonation nanodiamond. For testing the photothermal conversion performance of the detonation nanodiamond, $1 \mathrm{~mL}$ of aqueous DND dispersions was placed into a beaker and then irradiated with the sunlight of $100 \mathrm{~mW} \mathrm{~cm} \mathrm{~cm}^{-2}$ for $5 \mathrm{~min}$. The temperature variation of the aqueous ND dispersions was measured by an infrared thermometer at each time interval. Meanwhile, $1 \mathrm{~mL}$ of distilled water was used as a blank control group, and its temperature was assessed under the same condition.

2.3.2. Photothermal performance of hydrogel beads. Similarly, for testing the photothermal conversion performance of the composites, $1 \mathrm{~mL}$ of distilled water containing DND@SA hydrogel beads with different concentrations $\left(0-2 \mathrm{mg} \mathrm{mL}^{-1}\right)$ were placed into several cuvettes and then illuminated with the sunlight of $100 \mathrm{~mW} \mathrm{~cm}^{-2}$ for $20 \mathrm{~min}$, respectively. The change in temperature of the water containing DND@SA hydrogel beads was measured by an infrared thermometer under each time interval.

\subsection{Measurement of water adsorption characteristics}

2.4.1. Water adsorption. The swelling ratio of the DND@SA hydrogel beads was determined through the following steps. The dried samples (about $0.5 \mathrm{~g}$ ) were dipped entirely in $250 \mathrm{~mL}$ of distilled water at room temperature and soaked for $24 \mathrm{~h}$ to reach saturation. The swollen samples were filtered and weighted. The water adsorption (WA) was judged by the difference in weight of the samples at the beginning and at the time of swelling and calculated using the following formula: ${ }^{37}$ 
Table 1 Composition of the hydrogel beads for detonation nanodiamond and sodium alginate

\begin{tabular}{llll}
\hline $\begin{array}{l}\text { DND content in the } \\
\text { sample }\left(\mathrm{mg} \mathrm{mL}^{-1}\right)\end{array}$ & $\begin{array}{l}4 \mathrm{mg} \mathrm{mL} \mathrm{mL}^{-1} \mathrm{DND} \\
\text { suspension }(\mathrm{mL})\end{array}$ & $\begin{array}{l}\text { Sodium } \\
\text { alginate }(\mathrm{g})\end{array}$ & $\begin{array}{l}\text { Distilled } \\
\text { water }(\mathrm{mL})\end{array}$ \\
\hline 0 & 0 & 0.25 & 10 \\
0.4 & 1 & & 9 \\
0.8 & 2 & & 8 \\
1.2 & 3 & & 7 \\
2.0 & 5 &
\end{tabular}

$$
\mathrm{WA}\left(\mathrm{g} \mathrm{g}^{-1}\right)=\frac{W_{t}-W_{0}}{W_{0}}
$$

where $W_{0}(\mathrm{~g})$ and $W_{t}(\mathrm{~g})$ are the mass of the hydrogel beads at the dried state and at time $t$, respectively.

2.4.2. pH sensitivity. To determine the effect of $\mathrm{pH}$, solutions with $\mathrm{pH} 2-12$ were obtained by dilution with $\mathrm{HCl}$ or $\mathrm{NaOH}$, and the $\mathrm{pH}$ values were exactly measured with a $\mathrm{pH}$ meter. The $\mathrm{pH}$ sensitivity was studied by immersing the hydrogel beads in a solution of different $\mathrm{pH}$ values. In each case, the water adsorption was calculated from eqn (1).

\subsection{Sunlight-responsive test}

In order to further verify the sensitivity of the hydrogel beads to light, the sunlight-triggered water release experiments of the swelling-balanced hydrogel beads are necessary. $0.5 \mathrm{~g}$ of the dried hydrogel beads were placed in alkaline solution ( $\mathrm{pH} 9$ ), and after reaching an equilibrium swelling point, taken them out of the solution, and the surface water was absorbed by a filter paper, then weighed. The swollen hydrogel beads were placed under illumination and weighed after a certain period of time. The ability of the hydrogel beads to convert light into heat is judged by the change in water content, the water content (WT) was calculated using the following formula:

$$
\text { WT }\left(\mathrm{g} \mathrm{g}^{-1}\right)=\frac{W_{t}-W_{0}}{W_{0}}
$$

where $W_{0}(\mathrm{~g})$ and $W_{t}(\mathrm{~g})$ are the mass of the hydrogel beads at the dried state and at time $t$, respectively.

For a more intuitive understanding of the rise in temperature caused by DND under the illumination of sunlight, the above $0.8 \mathrm{mg} \mathrm{mL}$-1 of swelling-balanced DND@SA hydrogel beads were placed on a watch glass and then irradiated with the sunlight of $100 \mathrm{~mW} \mathrm{~cm}{ }^{-2}$ for $30 \mathrm{~min}$. The temperature rising process of the sample is captured by an infrared camera at different time intervals. Simultaneously, SA-Ca hydrogel beads used as a blank control group, and its temperature was estimated under the same condition.

\subsection{Characterization}

A Tecnaig2-20 TEM was used to observe the surface morphologies of detonation nanodiamond. A Hitachi S-4800 scanning electron microscope was used to observe their surface morphologies, and the samples were coated with gold using an Emitech K 550 Coater. The hardness of the samples was tested by a Universal TA texture analyzer. The functional groups of DND@SA hydrogel beads were confirmed using a PerkinElmer FTIR System 2000 in the $600-4000 \mathrm{~cm}^{-1}$ range at a resolution of $4 \mathrm{~cm}^{-1}$ using the $\mathrm{KBr}$ wafer technique. X-ray diffraction (XRD) analysis was conducted using diffractometer WJP75-91WJQ9. The absorption capacity of DND, SA-Ca, and DND@SA hydrogel beads to light were determined by Cary 5000 UV-VIS-NIR spectrophotometer. Thermogravitational analysis (TGA) was carried out on a HENVEN HCT-3 by heating the samples to $900{ }^{\circ} \mathrm{C}$ under a nitrogen atmosphere. All the samples were completely dry before characterization. Visible light irradiation was provided by exposing samples to light from an analog daylight lamp source (PLS-SXE300C), which yielded an intensity of $100 \mathrm{~mW} \mathrm{~cm}^{-2}$, as measured by an FZ-A Radiometer. The temperature variation of DND@SA composites aqueous solution was monitored using a non-contact infrared thermometer (GM320, BENETECH). An infrared camera (CHAUVIN ARNOUX/ CA73) was used to take infrared photographs.

\section{Results and discussion}

\subsection{Preparation and characterization of DND@SA hydrogel beads}

In the current work, the fabrication of the DND@SA hydrogel beads began with the synthesis of DND-SA suspensions. The detailed fabrication process of the DND@SA hydrogel beads is schematically illustrated in scheme 1. The sonicated DND suspensions were mixed with the SA in advance to obtain DNDSA mixed suspensions (step 1). Then, the mixed suspensions were dropped into the calcium chloride solution, at a constant injection rate using a syringe pump. Once the spherical droplet of the DND-SA suspensions reached the calcium chloride solution, calcium ions immediately diffused into the droplet of the mixed solution. At this moment, negatively charged $-\mathrm{COO}^{-}$ groups on the surface of SA were crosslinked with positively charged $\mathrm{Ca}$ (II) ions, forming a solidified Ca-alginate bead (step 2). The continuous dropping of DND-SA suspension also induced the simultaneous formation of solidified Ca-alginate in the droplet, producing spherical Ca-alginate hydrogels containing DND nano-particles. The interaction between the nanodiamond and substrates maybe include covalent ${ }^{38}$ and non-covalent interactions, ${ }^{39}$ like hydrogen bonding interactions and electrostatic interactions. Similarly, the oxygen- and nitrogen-groups adsorbed on the surface of the DND also form a possible hydrogen bond with the hydroxyl and carboxyl groups on the SA molecular chain. In this way, the outstanding properties from the DND were assembled into the skeleton of the SA matrix, and thus take advantage of the excellent photothermal properties of DND.

Predicting from their structure, the as-prepared DND@SA photothermal hydrogel beads possess a sterling ability to harvest light energy and sustained water release due to their denser hydrophilic crosslinked network structure, which is helpful for the water adsorption in potential agricultural applications. Specifically, due to the abundant hydrophilic groups on the surface of SA and within the internal threedimensional network of the polymer matrix, the hydrogel 
Sodium alginate

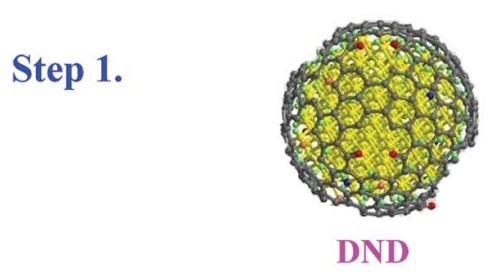

Detonation nanodiamond

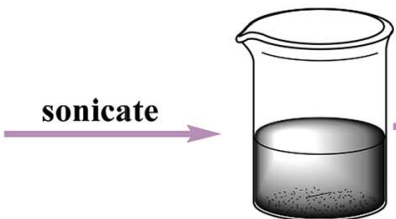

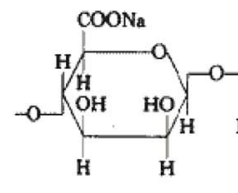

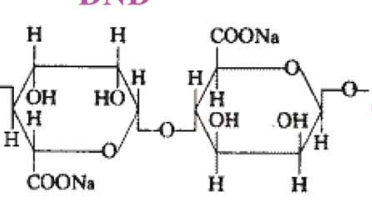

SA

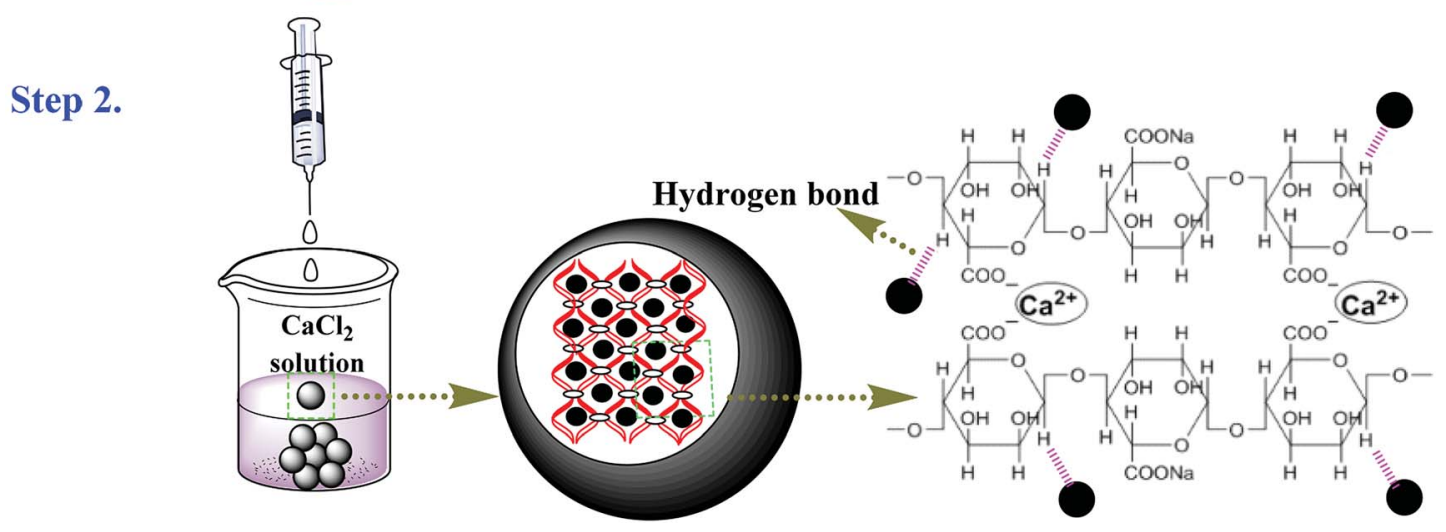

Scheme 1 Proposed mechanism for the formation of DND@SA hydrogel beads.

beads firstly have exceptional water adsorption capacity through strong hydrogen bond interaction. Secondly, the addition of DND not only imparts the photothermal properties of the hydrogel beads but also enhances the adsorption capacity for water owing to the luxuriant hydrophilic group of DND. ${ }^{40} \mathrm{At}$ the same time, the internal polymer matrix can firmly fix the DND in the stent to improve the dispersion of DND. Moreover, the DND with super-hard properties as a filler into the SA matrix significantly has improved the mechanical property of the hydrogel beads. Namely, the hardness of the DND@SA composites is much higher than SA-Ca and increases with the increase of DND content, as shown in Fig. S1. $\dagger$ From these points of view, the formed DND@SA hydrogel beads combine the synergistically photothermal property of DND and hydrophilic of SA matrix. Such remarkable properties have opened an appropriate route for controlled release of water.

To intuitively illustrate the above-mentioned formation procedures, the optical images of samples were recorded. The results are shown in Fig. 1a. It can be seen that the color of the beads turned from nearly milky white to gray, to black as the amount of DND increased gradually. Such visual inspection suggests that the package of DND nanoparticles within the SA beads have been fulfilled. In addition, it can be found that both SA-Ca and DND@SA hydrogel beads had no significant difference in size. In another word, the DND@SA hydrogel beads had an almost equivalent diameter to that of the parallel SA-Ca hydrogel beads, which are all in the range of $2.5-3.0 \mathrm{~mm}$ and an average value of about $2.7 \mathrm{~mm}$. TEM analysis in Fig. 1b shows that the raw DND particles synthesized by detonation were close to spherical and contained single-crystal grains of a size ranging from $10 \pm 5 \mathrm{~nm}$. From Fig. $\mathrm{S} 2, \dagger$ it can be seen that the primitive DND particles have a large tendency to become agglomerated. To observe the dispersion of DND particles on the surface of samples, the surface characteristics of DND@SA hydrogel beads were examined at high magnification by SEM. As shown in Fig. 1c and d, the DND@SA hydrogel beads had a relatively small size compared to pure SA beads under the same drying conditions, which is attributed to the high thermal conductivity of detonation nanodiamond, making the drying of composite hydrogel beads more complete. The surface of the beads after drying in an oven became rough and exhibited many wrinkles compared with the naked SA hydrogel beads, which are beneficial to the water adsorption and release behaviors from the beads. Moreover, lots of small burs were visible on the surface of samples, and a few of cracks of the gold coating appeared occasionally on the surface of both beads, due to the volume shrinkage after drying. Through a larger magnification observation of samples in Fig. 1e and f, some uniform and orderly folds are clearly visible on the surface SA-Ca bead samples (Fig. 1e), whereas in the composite DND@SA ones (Fig. 1f) these features were absent and the surface grains smaller, providing an overall more compact surface. The caused 


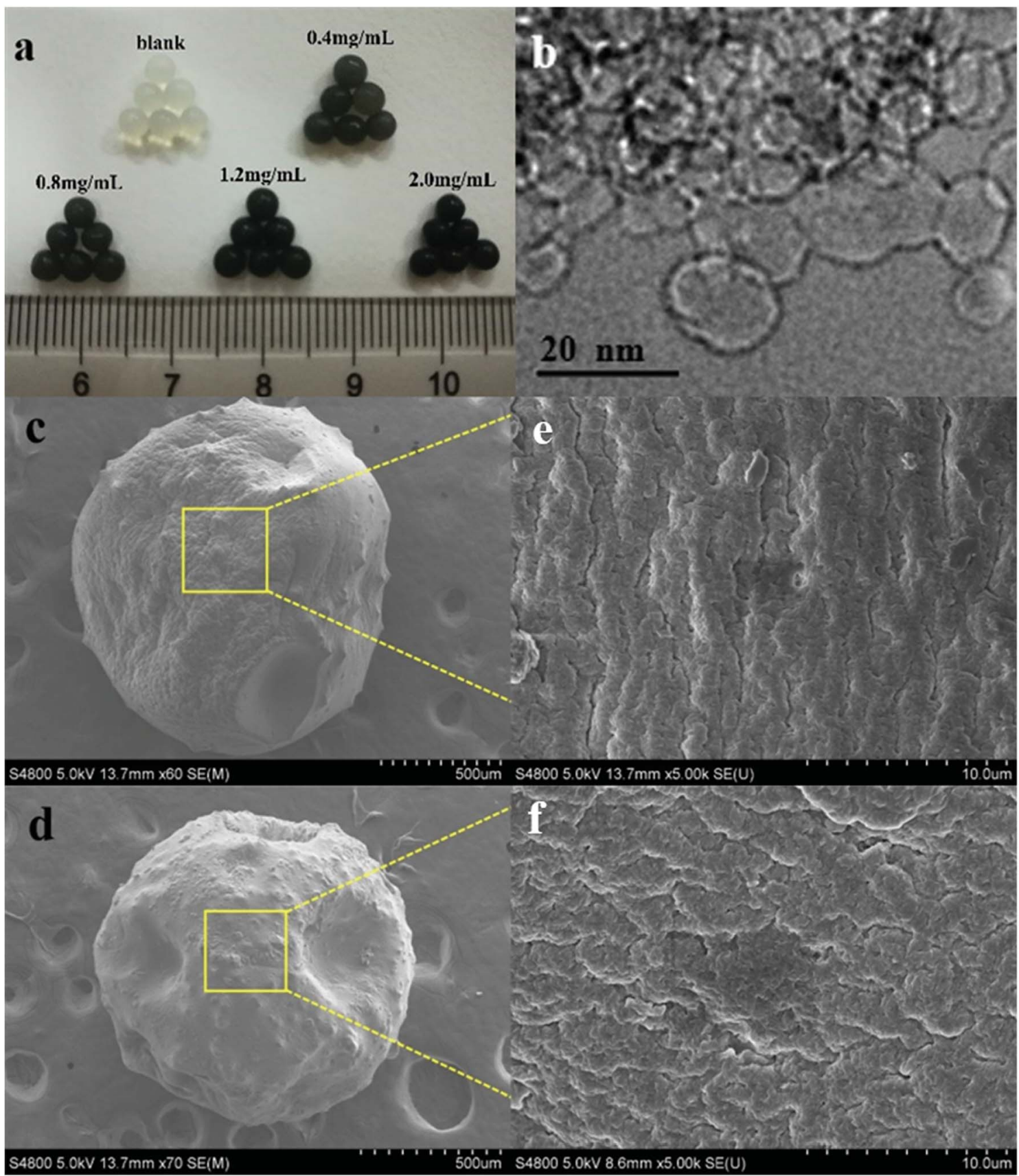

Fig. 1 The appearance of DNDQSA hydrogel beads with different DND content (a) and TEM images of DND (b) and SEM images of SA-Ca beads (c and e) and DND@SA hydrogel beads ( $d$ and f).

reason was that the addition of DND could form a tighter threedimensional network for the polymer matrix and consequently intensify the denser cross-linked on the surface of the beads. Furthermore, there are no agglomerates of DND on the surface of DND@SA composite beads, which hints that DND was dispersed uniformly in the polymer matrix.

To validate the interaction force between DND nanoparticles and SA beads, Fourier transform infrared (FTIR) spectra was employed to characterize the structure of intermediates and the final products. The FTIR spectra of DND, SA, SA-Ca beads and DND@SA are exhibited in Fig. 2a. In the spectrum of DND, the peak at $3420 \mathrm{~cm}^{-1}$ is the $-\mathrm{OH}$ stretching vibration absorption peak, indicating that the surface of DND is rich in -OH. Bending vibration absorption peak of adsorbed water molecules is observed at $1630 \mathrm{~cm}^{-1}$. $^{41}$ The absorbance peak at $1110 \mathrm{~cm}^{-1}$ is attributed to the $\mathrm{C}-\mathrm{C}$ stretching vibration of graphite. ${ }^{42} \mathrm{SA}$ showed a broad peak at $3437 \mathrm{~cm}^{-1}$ for its hydrogen-bonded $\mathrm{OH}$ group. Asymmetric and symmetric stretching of the $\mathrm{C}=\mathrm{O}$ group of alginate is observed at 1642 and $1401 \mathrm{~cm}^{-1}$, respectively. ${ }^{43} \mathrm{In}$ addition, sodium alginate showed a characteristic peak at 1096 and $820 \mathrm{~cm}^{-1}$ corresponding to $\mathrm{C}-\mathrm{O}$ stretching vibration and $\mathrm{Na}-\mathrm{O}$ bond vibration, this indicates that SA has a large number 
of random coil structures. ${ }^{44}$ For the FTIR curve of SA-Ca, the cross-linking process with $\mathrm{Ca}^{2+}$ gave rise to an apparent shift of $-\mathrm{COO}^{-}$asymmetric stretching vibration from 1642 to $1646 \mathrm{~cm}^{-1}$ and a synchronously $-\mathrm{COO}^{-}$symmetric stretching vibration from 1401 to $1421 \mathrm{~cm}^{-1}$, showing the formation of a SA-Ca hydrogel by ionic bonding between carboxyl groups of SA and $\mathrm{Ca}^{2+} \cdot{ }^{45}$ FTIR spectrum of the DND@SA composites shows that no new bonds were formed compared with SA-Ca and the absorption peaks of the DND were overlapped by the strong absorption peaks of SA. Obviously, it was observed a slight red shift of the stretching vibration peaks from $3425 \mathrm{~cm}^{-1}$ in the SA-Ca and $3420 \mathrm{~cm}^{-1}$ in DND to $3415 \mathrm{~cm}^{-1}$ in the DND@SA composites. This change confirms that the interaction between DND and the SA is based on hydrogen bonding rather than covalent interactions.

XRD analysis was provided to study the crystallinity of detonation nanodiamond, sodium alginate, SA-Ca beads, and DND@SA hydrogel beads, and the results are shown in Fig. $2 \mathrm{~b}$. The peak at $2 \theta$ of $25^{\circ}$ corresponds to the (002) crystal plane of graphite. The (111) and (220) peaks are characteristic of detonation-synthesized diamond particles. ${ }^{\mathbf{4 6}}$ Sodium alginate shows several crystalline peaks at $2 \theta$ of 13.5 , and $21.9^{\circ}$ due to hydrogen bonding among its hydroxyl groups, as also reported elsewhere. ${ }^{47}$ From the XRD pattern of calcium alginate beads, we can see two major peaks at $2 \theta$ diffraction angles of 13 and $40^{\circ}$. Characteristic peaks for calcium alginate are known to appear at angles of $13^{\circ}, 56^{\circ}, 20.64^{\circ}, 20.06^{\circ}, 28.96^{\circ}$, and $36.40^{\circ}$, thus demonstrating its semi-crystalline nature. ${ }^{48}$ The DND@SA composites showed two broad peaks at $2 \theta$ of $22^{\circ}$, and $39^{\circ}$ as observed in Fig. 2b. It is worth noting that low DND content is responsible for the absence of the diffraction peak of DND. ${ }^{49}$ Meanwhile, the SA-Ca structure was not significantly affected by the incorporation of DND indicating that there was mainly physical interaction between SA and DND. ${ }^{50}$

Owing to the high thermal conductivity of primitive nanodiamond, ${ }^{51}$ introductions of DND into SA-Ca hydrogel inevitably have effects on the thermal stability of composite hydrogel. Thus, the thermal decomposition process of both SA-Ca and DND@SA composites was conducted, and the results are displayed in Fig. 2c. As it is shown, the thermal decomposition process of both SA-Ca and DND@SA composites with DND content of $0.8 \mathrm{mg} \mathrm{mL}^{-1}$ was rather similar. The TGA profiles of both types of beads can be analyzed within three steps: the first step occurring in the range of $25-200{ }^{\circ} \mathrm{C}$, the second occurring in the range of $200-400{ }^{\circ} \mathrm{C}$, and the last occurring in the range of $400-700{ }^{\circ} \mathrm{C}$. The first step accounts for about $15 \%$ of weight loss, which can be attributed to the desorption of physically adsorbed water and removal of structural water, thus indicating excellent water adsorption characteristics of both types of beads. ${ }^{48,52}$ The second step, which is the rapid weight loss stage, corresponds to the thermal cracking processes of some functional groups (such as $-\mathrm{OH}$ and $-\mathrm{COOH}$, etc.) and cation
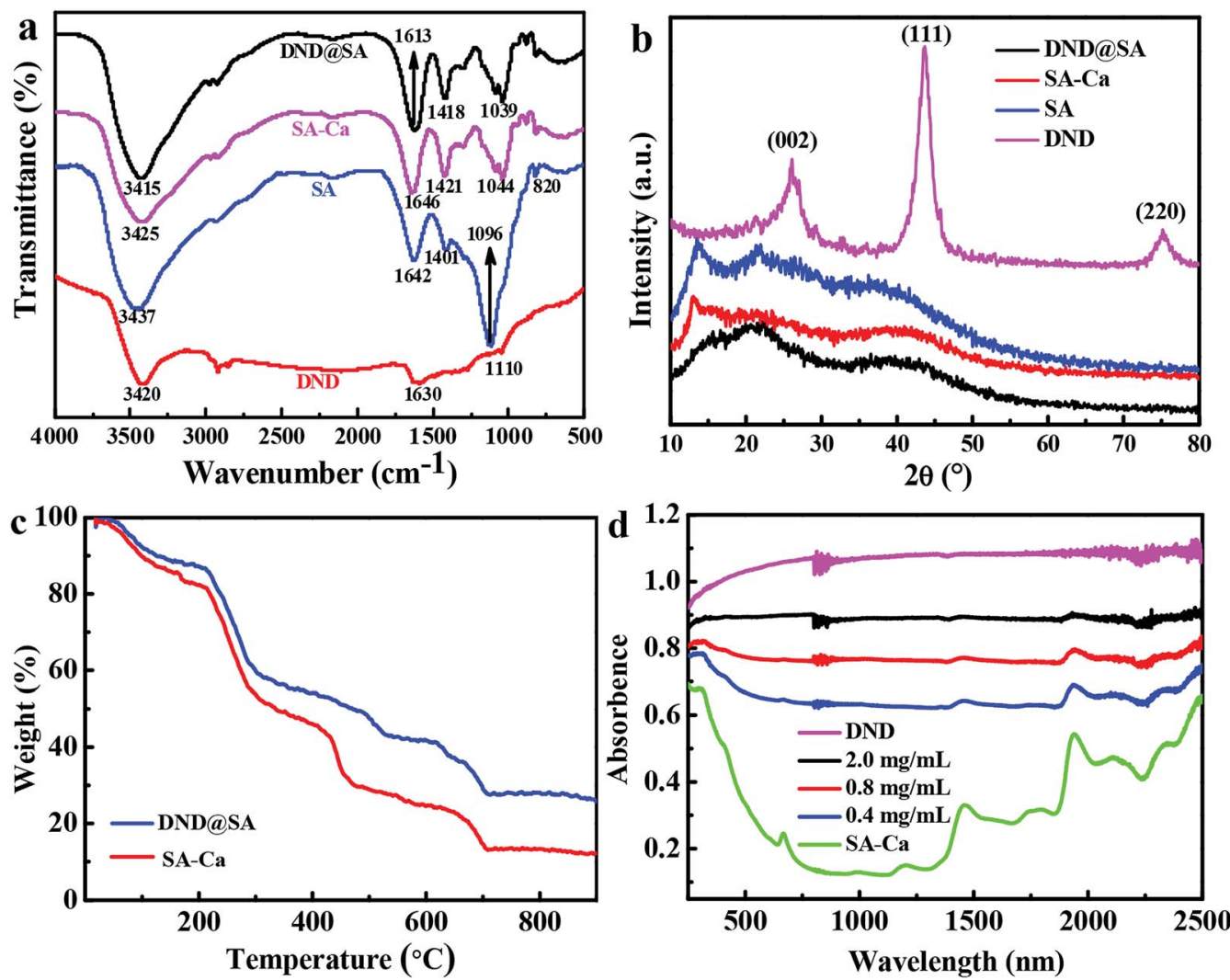

Fig. 2 (a) FTIR spectra, (b) XRD of DND, SA, SA-Ca beads, and DNDaSA hydrogel beads. (c) TGA curves of SA-Ca beads and DNDaSA hydrogel beads in a nitrogen atmosphere. (d) UV-vis diffuse reflectance spectra of DND, SA-Ca beads, and various concentrations of DND $a S A$ hydrogel beads. 
bonding bridges, the volatilization of small molecular substances, and the formation of carbonized products, while the bottom to the further decomposition of carbonized products and the removal of residual volatile components. Nanodiamonds are well-known to be thermally stable. Compared to DND@SA beads with a weight remaining of $\sim 29 \%$, the residue mass of SA-Ca beads was found to be low to $\sim 11 \%$ within the same temperature frame, which results from the decomposition of alginic molecular chain and the basic skeleton. ${ }^{53,54}$ In brief, it can be demonstrated the relatively excellent thermal stability of the DND@SA hydrogel due to the introduction of DND, which is beneficial to the water release under irradiation through thermal conductivity of DND.

The photothermal conversion capability was closely related to their optical absorption properties of DND. To confirm the light absorption of obtained samples, the UV-vis diffuse reflectance spectra of DND, SA-Ca beads, and various concentration of DND@SA hydrogel beads were conducted, and the results are showed in Fig. 2d. It can be seen that the raw DND exhibits a high absorption capacity for the light from ultraviolet to nearinfrared, which can act as an efficient light energy absorber, while SA-Ca beads show comparatively limited light absorption ability. Furthermore, compared with SA-Ca beads, the absorbance of DND@SA hydrogel beads with $0.4 \mathrm{mg} \mathrm{mL}{ }^{-1} \mathrm{DND}$ content has been significantly extended to visible and nearinfrared regions, and the absorption is proportional to the content of DND. Such enhanced light absorption allows DND@SA hydrogel beads to be more active than SA-Ca beads, which lays foundations for efficient water release through their photothermal performance.

\subsection{Photothermal performance of DND@SA hydrogel beads}

The photothermal conversion capability of DND nanoparticles under light irradiation was evaluated to validate their potential as sunlight-responsive materials. As shown in Fig. 3a, the pure water did not undergo a significant temperature change upon irradiation with the sunlight of $100 \mathrm{~mW} \mathrm{~cm}^{-2}$ for $5 \mathrm{~min}$, while the temperature of the $0.8 \mathrm{mg} \mathrm{mL}^{-1}$ aqueous dispersion of DND rose from room temperature to $31.4{ }^{\circ} \mathrm{C}$ over $3 \mathrm{~min}$. Compared with distilled water, the inner wall of the cuvette containing DND showed obvious water droplets after 5 min irradiation (the inset image of Fig. 3a). These results indicate that DND nanoparticles produced enough heat under sunlight irradiation to cause a substantial temperature increase of aqueous DND dispersions, confirming that the DND can convert the photon energy into thermal energy.

To quantitatively determine whether the amount of generated heat depends on the concentration of DND, the temperature variations of DND@SA hydrogel beads with different DND concentrations placed in distilled water under irradiation with sunlight were investigated. The results showed in Fig. 3b revealed that the temperature of all the aqueous containing DND@SA hydrogel beads increased gradually with exposure time, while the aqueous containing SA-Ca beads showed a smaller temperature change under identical illumination conditions. This phenomenon indicates that the DND@SA efficiently converts the photon energy into heat energy by absorbing light and produces enough heat to cause a significant temperature increase, confirming that the photothermal conversion of DND@SA hydrogel beads could be induced by sunlight. In addition, DND@SA hydrogel beads played a great influence on the temperature of the surrounding medium, which increased monotonically with the stagewise increase of the DND concentration. For the mixture containing DND@SA hydrogel beads at concentrations ranging from 0.4 to $2.0 \mathrm{mg}$ $\mathrm{mL}^{-1}$ illuminated for $20 \mathrm{~min}$, the temperature increment varied from 14.6 to $21.1{ }^{\circ} \mathrm{C}$ (Fig. $\mathrm{S} 3 \dagger$ ). These results mean that the concentration of DND and exposure time control the degree of temperature increase and can be tailored for a specific application. Therefore, it can be expected from the results that DND@SA hydrogel beads have significant potential as a sunlight-responsive release system to trigger the release of the loaded water with the utilization of detonation nanodiamond photothermal agent.

\subsection{Water adsorption of the DND@SA hydrogel beads}

3.3.1. Water adsorption of SA-Ca and DND@SA hydrogel beads. Water adsorption is one of the biggest advantages of a hydrogel, i.e., the application of a composite hydrogel depends largely on its water-adsorbing capacity. ${ }^{55,56}$ As can be seen from Fig. 4a, in the earlier swelling stage, the water adsorption of the hydrogel beads increased rapidly. Subsequently, the water adsorption increased slowly. The reason for this result is assigned to that the water molecule at the earlier swelling stage has great osmotic pressure, facilitating the infiltration of the water molecules into the interior of hydrogel beads and boosted the swelling rate. Nevertheless, with the continuation of the swelling process, more and more water permeated into the interior of the hydrogel beads, and the osmotic pressure of the hydrogel beads reduces, hence causing the decrease of the swelling rate with time. Furthermore, we found that the water adsorption rate of the pure SA hydrogel beads was significantly influenced by its particles size. The higher water adsorption rate is primarily observed in the hydrogel beads with small particles size, and hence, the time needed to reach equilibrium adsorption was less compared with the large ones. However, the amount of water adsorbed by the hydrogel beads of different particle size at final equilibrium adsorption was not significantly influenced by hydrogel particles size. Actually, the large particle possesses a small specific surface area (SSA) while the small ones possess large SSA. ${ }^{57,58}$ The higher water adsorption capacity of the small particles relative to large ones might be attributed to the higher SSA of the small particles. The high SSA enhanced the capability of the small particles to adsorb and retain water, as its contact area with water molecules was much higher than the large particles. In addition, Fig. 4a shows that the hydrogel beads with various DND contents exhibited different water adsorption in deionized water. The water adsorption of DND@SA hydrogel beads in deionized water raised with increasing detonation nanodiamond content, reaching maximum adsorption about $1.65 \mathrm{~g}$ $\mathrm{g}^{-1}$ at a content of $0.8 \mathrm{mg} \mathrm{mL}^{-1}$ and then falling off. This is 

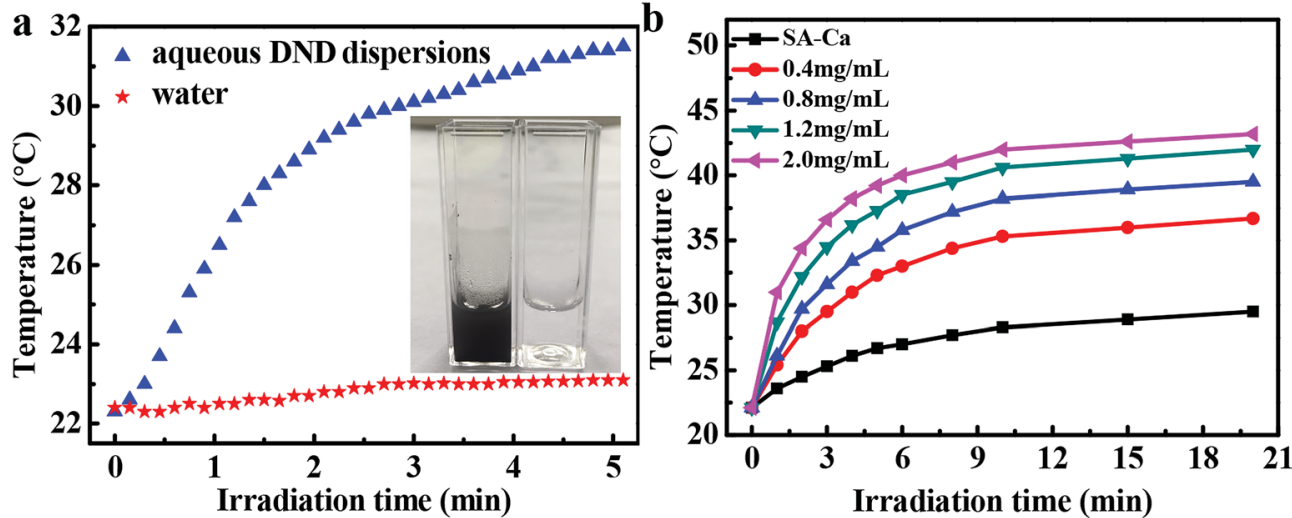

Fig. 3 (a) Temperature changes of pure water and $0.8 \mathrm{mg} \mathrm{mL}^{-1}$ aqueous DND dispersions under irradiation with the sunlight of $100 \mathrm{~mW} \mathrm{~cm}^{-2}$. (Inset) Photograph of aqueous DND dispersions and distilled water after 5 min of irradiation. (b) Temperature changes of distilled water containing DNDCSA hydrogel beads with different DND concentrations under irradiation with the sunlight of $100 \mathrm{~mW} \mathrm{~cm}^{-2}$.

because, as the amount of DND increased from a content of $0.4 \mathrm{mg} \mathrm{mL}^{-1}$ to $0.8 \mathrm{mg} \mathrm{mL}^{-1}$, there are many hydrophilic sites provided by the surface functional groups and micropores of the graphitic shell of DND, ${ }^{38,40}$ which can increase the wateradsorbing capacity of composites. Fig. S4 $\uparrow$ clearly presents the excellent stability of hydrophilicity for the DND. With further increased content of DND, the water adsorption showed a declining trend. The swelling behavior of the hydrogel beads primarily depends on the amorphous region in the composites to adsorb free water or form hydrogen bonds between water molecules and polymer matrix. ${ }^{59}$ On the one hand, the increase of detonation nanodiamond content in the hydrogel beads leads to increased spatial resistance and reduced micropores. On the other hand, the number of hydrogen bonds between detonation nanodiamond and SA increases as the DND content rises. As shown in Fig. $\mathbf{S 5 , \dagger}$ these results improve the crystalline
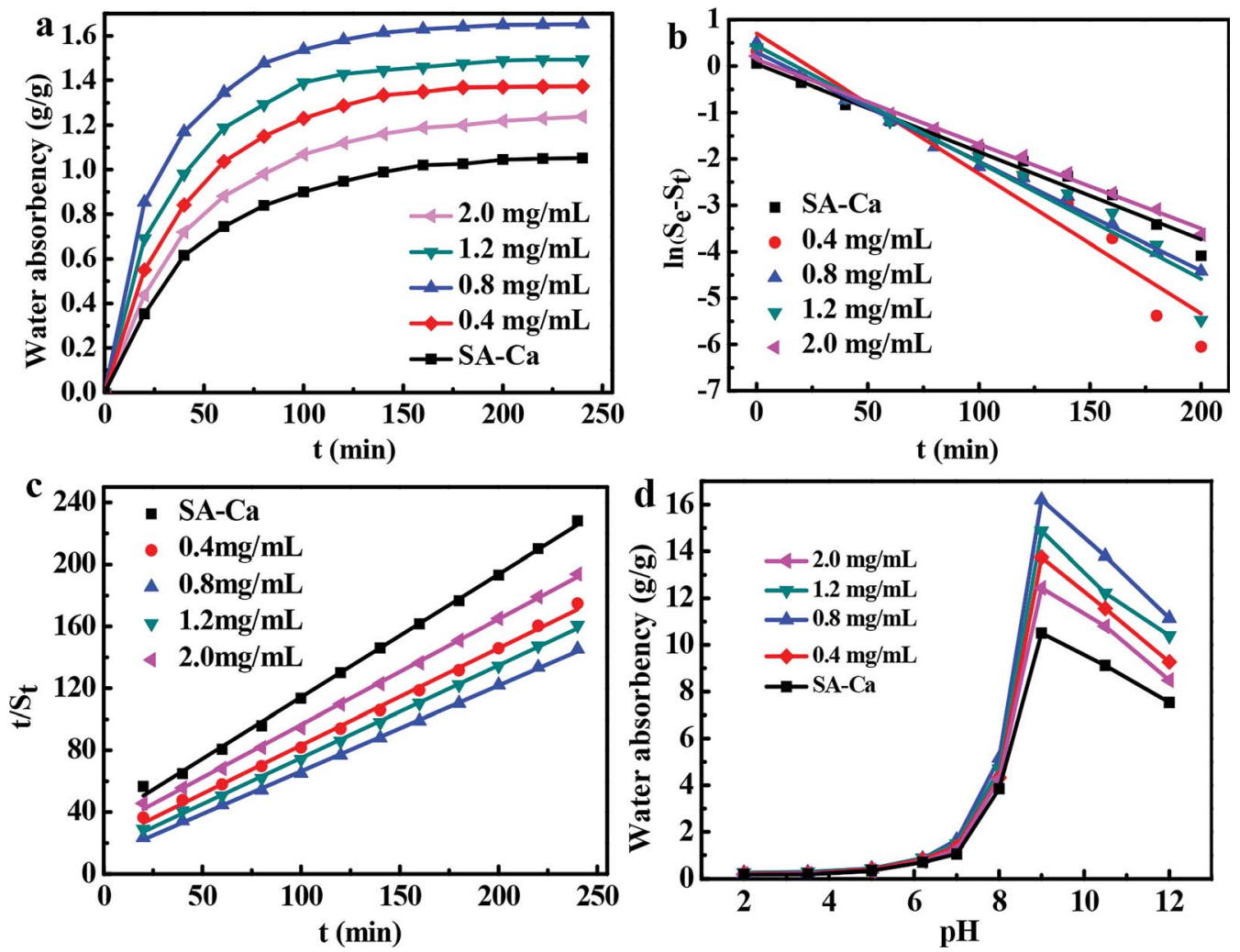

Fig. 4 (a) Water adsorption of SA-Ca and DNDQSA hydrogel beads with different amounts of DND, (b) pseudo-first-order kinetic model, (c) pseudo-second-order kinetic model. (d) Effect of $\mathrm{pH}$ on the swelling behavior of SA-Ca and DND@SA hydrogel beads with different amounts of DND. 
areas and reduce the amorphous region as well as the free hydroxyl group in the hydrogel beads. Ultimately, the values of water uptake decreased.

Analysis of the swelling kinetics is beneficial to clarify the mechanism of the swelling process. Thus, pseudo-first and pseudo-second-order kinetic models were used to fit the experimental data to evaluate the water adsorption efficiency of the DND@SA hydrogel beads. The models are expressed in the following formulas: ${ }^{60,61}$

$$
\begin{gathered}
\ln \left(S_{\mathrm{e}}-S_{t}\right)=\ln S_{\mathrm{e}}-K_{1} t \\
\frac{t}{S_{t}}=\frac{1}{K_{2} S_{\mathrm{e}}^{2}}+\frac{t}{S_{\mathrm{e}}}
\end{gathered}
$$

where $S_{t}\left(\mathrm{~g} \mathrm{~g}^{-1}\right)$ and $S_{\mathrm{e}}\left(\mathrm{g} \mathrm{g}^{-1}\right)$ stand for the water adsorption at contact time $t(\mathrm{~min})$ and the equilibrium state, and $K_{1}\left(\mathrm{~min}^{-1}\right)$ and $K_{2}\left(\mathrm{~g} \mathrm{~g}^{-1} \mathrm{~min}^{-1}\right)$ are the rate constants of the pseudo-first and pseudo-second-order kinetic models, respectively. The fitting curves, kinetic parameters, and correlation coefficients are presented in Fig. $4 \mathrm{~b}, \mathrm{c}$ and Table S1. $\dagger$ The $S_{\mathrm{e} \text {, cal }}$ values as obtained from the pseudo-second-order model were relatively near to the data obtained experimentally $\left(S_{\mathrm{e}}\right.$, exp $)$ than those from the pseudo-first-order model. Moreover, the correlation coefficient $\left(R^{2}\right)$ of the pseudo-second-order model is much closer to 1.0, indicating that the pseudo-second-order kinetic model was applicable to the swelling process of DND@SA hydrogel beads.

3.3.2. $\quad$ pH-sensitive water adsorption behavior of DND@SA hydrogel beads. In order to determine whether composite DND@SA beads possess $\mathrm{pH}$ sensibility, change on water adsorption was observed applying different buffer solutions. Fig. 4d shows the water adsorption property of SA-Ca and DND@SA hydrogel beads in solutions with different $\mathrm{pH}$ values regulated by diluting with $\mathrm{HCl}$ or $\mathrm{NaOH}$ solutions. It can be found from Fig. $4 \mathrm{~d}$ that the water adsorption depended on the $\mathrm{pH}$ of the swelling medium. The water adsorption initially kept almost invariant in the $\mathrm{pH}$ ranged $2-5$, then increased with increasing $\mathrm{pH}$ to 9 and decreased with increasing $\mathrm{pH}$ from 10 to 12. The water adsorption of hydrogel beads rose as the DND content increased from 0 to $0.8 \mathrm{mg} \mathrm{mL}^{-1}$, and then reduced until the DND content increased to $2 \mathrm{mg} \mathrm{mL}^{-1}$. The maximum water adsorption capacity $\left(16.20 \mathrm{~g} \mathrm{~g}^{-1}\right)$ was achieved at $\mathrm{pH} 9$ with $0.8 \mathrm{mg} \mathrm{mL}^{-1}$ DND contents. This is because the sodium alginate molecular chain is composed of mannuronic acid and guluronic acid, and their $\mathrm{p} K_{\mathrm{a}}$ values are 3.38 and 3.65 , respectively. Once the $\mathrm{pH}$ value of the solution was changed, the carboxyl group of the molecular chain of sodium alginate would change accordingly, thereby affecting the swelling rate of the hydrogel. At $\mathrm{pH}<6$, most carboxylate groups $\left(-\mathrm{COO}^{-}\right)$immobilized on the sodium alginate chain could combine with hydrogen ions to form carboxyl groups, which weaken the electrostatic repulsion among the anionic groups, thereby preventing the expansion of the polymer matrix. At $7<\mathrm{pH}<9$, more carboxylate groups were ionized, and the number of negatively charged $-\mathrm{COO}^{-}$groups increased, which enhanced the repulsive force. ${ }^{62,63}$ Consequently, the hydrogel beads were tended to swell more in this $\mathrm{pH}$ range. However, when the $\mathrm{pH}$ was higher than 9, the effective repulsive force between the carboxylic acid anions is weakened due to the electrostatic attraction of $\mathrm{Ca}^{2+}$ and other counter ions, so that the polymer chain shrinks. As a result, the swelling rate of the hydrogel showed a significant decrease from $\mathrm{pH}$ 9. At neutral $\mathrm{pH}$, the water adsorption of hydrogel beads with different DND contents was in the following order: $0.8 \mathrm{mg} \mathrm{mL}^{-1}>1.2 \mathrm{mg} \mathrm{mL}^{-1}>0.4 \mathrm{mg} \mathrm{mL}^{-1}>$ $2.0 \mathrm{mg} \mathrm{mL} L^{-1}>0.0 \mathrm{mg} \mathrm{mL}^{-1}$, which was consistent with the result in distilled water.

\subsection{Sunlight-triggered water release of the DND@SA hydrogel beads}

By combining the sodium alginate hydrogel and the photothermal sensitive detonation nanodiamond, the DND@SA hydrogel beads were obviously endowed with water holding capacity and tunable water release property under sunlight irradiation. Thus, to illustrate the effect of light intensities on water release, we performed a sunlight-triggered water release test on the swollen-balanced gel beads using an analog daylight lamp source. In this section, the hydrogel beads which reached a swelling equilibrium in an alkaline solution at $\mathrm{pH} 9$ were used, in consideration of their maximum water adsorption.

Fig. 5a shows the change of water content of hydrogel beads with different amounts of DND after 10 minutes of visible light illumination. We found that the sunlight-triggered water release property can be customized by modulating the amount of DND, as well as the irradiation time. As showed in Fig. 5a, a higher loading of DND induced a greater water release under identical conditions. In contrast, pure SA-Ca beads undergo a smaller change in water content, indicating that DND can absorb sunlight and convert it into heat, thereby resulting in water release from the hydrogel beads.

For evaluating the effect of light intensities on water release, we further examined the water content changes of the hydrogel beads under sunlight irradiation at different light intensities. As seen in Fig. 5b, DND@SA hydrogel beads containing $0.8 \mathrm{mg}$ $\mathrm{mL}^{-1}$ DND in the alkaline solution $(\mathrm{pH}$ 9) showed a swelling ratio of 16.20. When exposed to sunlight, the DND@SA hydrogel beads underwent significant water release proportional to the exposure time. For the sample under sunlight irradiation for $10 \mathrm{~min}$ at a light intensity of $50 \mathrm{~mW} \mathrm{~cm}{ }^{-2}$, the water content decreased to 13.47, while the water content of the same sample was 10.90 after irradiation at a light intensity of $100 \mathrm{~mW} \mathrm{~cm}^{-2}$. Irradiation with $150 \mathrm{~mW} \mathrm{~cm}^{-2}$ sunlight for $10 \mathrm{~min}$ led to the decrease of the water content to 7.81, which corresponds to an approximately $51 \%$ decrease in the weight compared to the initial equilibrium state before light exposure. These results suggest that the degree of water release of DND@SA hydrogel beads can be easily manipulated by controlling the light intensities.

To identify that photothermal conversion by DND is responsible for the sunlight-triggered water release of the hydrogel beads, we attempted to take thermal images of the composite hydrogel beads under irradiation conditions. As seen in Fig. 5c, the surface temperature of the SA-Ca and DND@SA was maintained at around $21.5{ }^{\circ} \mathrm{C}$ at the equilibrium swelling 

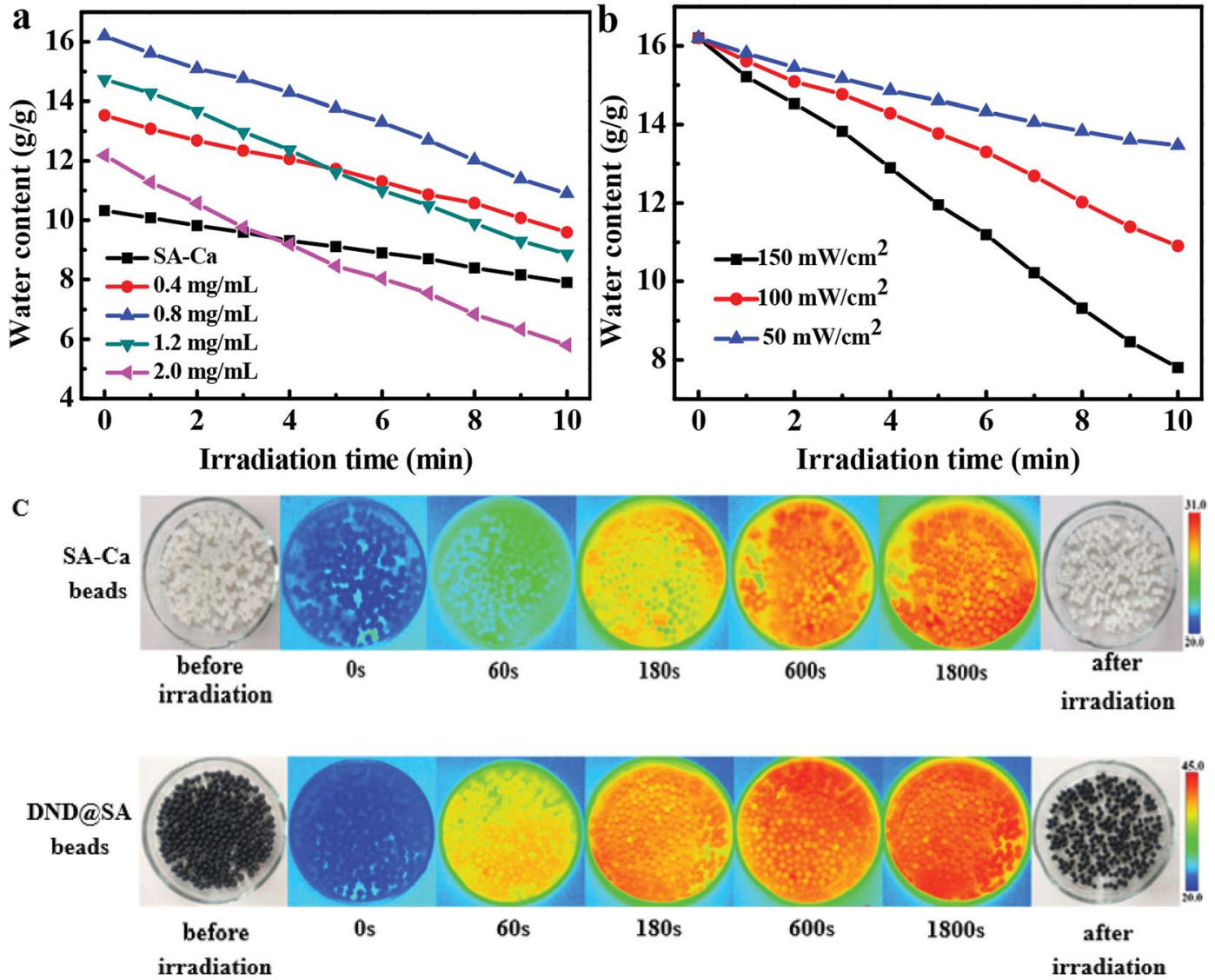

Fig. 5 (a) Water content for SA-Ca and DND@SA hydrogel beads with different amounts of DND exposed to $100 \mathrm{~mW} \mathrm{~cm}^{-2}$ of sunlight for $10 \mathrm{~min}$. (b) Water content for DND CSA hydrogel beads containing $0.8 \mathrm{mg} \mathrm{mL}^{-1}$ DND exposed to 50, 100, and $150 \mathrm{~mW} \mathrm{~cm}^{-2}$ of sunlight for $10 \mathrm{~min}$. (c) Infrared photos of the SA-Ca and $0.8 \mathrm{mg} \mathrm{mL}^{-1}$ of DNDQSA hydrogel beads. The photos, in order from left to right, correspond to $t=0,60,180$, 600 , and $1800 \mathrm{~s}$ after irradiation with the sunlight of $100 \mathrm{~mW} \mathrm{~cm}^{-2}$.

state. Once the light was turned on, the temperatures of the surface of the bead increased sharply in the initial $600 \mathrm{~s}$ and rose smoothly afterward. After $30 \mathrm{~min}$ illumination, the maximum temperature of the surfaces in SA-Ca and DND@SA hydrogel beads reached $\sim 30.5$ and $44.6{ }^{\circ} \mathrm{C}$, respectively. Due to the outstanding light absorption capability imparted by the

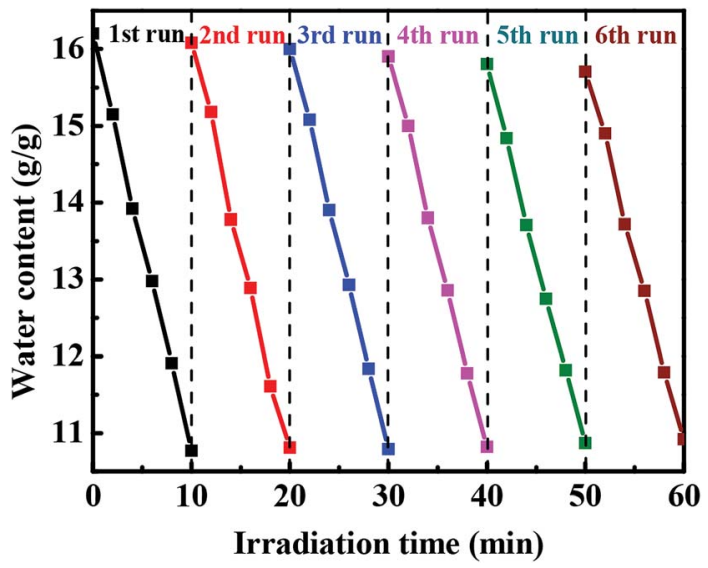

Fig. 6 Cycle test curves for sunlight-triggered water release of DNDASA hydrogel beads containing $0.8 \mathrm{mg} \mathrm{mL}^{-1}$ DND. Each cycle consists of light irradiation for 10 min followed by a re-swelling process. detonation nanodiamond, the DND@SA hydrogel beads exhibited a much higher maximum steady temperature than SACa beads under the same illumination, demonstrating that the DND can effectively absorb sunlight to heat the hydrogel matrix.

To estimate the reuse stability of the composite beads, the cycle test of DND@SA hydrogel beads was performed six times. In Fig. 6, we found that the water release of the hydrogel beads triggered by sunlight could be repeated multiple times without significant variation. The water contents were measured at certain intervals for 10 min irradiation, soon after turning the sunlight off, the DND@SA hydrogel beads were placed in alkaline solution at $\mathrm{pH} 9$ to reach the equilibrium swelling point. As showed in Fig. 6, the hydrogel beads exhibited reversible changes in water content in each cycle with constant and reliable water content changes. After six cycles, the hydrogel beads still had better responsivity, indicating the excellent reversibility of DND@SA hydrogel beads can significantly meet the long service life requirements for their potential applications in agricultural release systems.

\section{Conclusion}

In summary, a novel DND@SA hydrogel bead that can be used as a sunlight-controlled release-targeted system for water was 
synthesized using a simple conventional method, ion gelation. The composites were characterized by various advanced techniques to estimate structural and photothermal properties. SEM observation shows that DND nanoparticles in the hydrogel beads have good affinity with the SA matrix and disperse uniformly into the SA matrix. FTIR and XRD indicate that DND nanoparticles with abundant functional groups on the surface interact with the SA matrix. The resultant product with $0.8 \mathrm{mg}$ $\mathrm{mL}^{-1}$ DND content achieved a maximum water-adsorption of $1.65 \mathrm{~g} \mathrm{~g}^{-1}$ in distilled water. The pseudo-second-order kinetic model could fit better than a pseudo-first-order kinetic model to the swelling process of DND@SA hydrogel beads in distilled water. As a result of its particular photothermal performance and functional groups, hydrogel beads were significantly sensitive to the sunlight and the $\mathrm{pH}$ value of the external solution and showed varying water release effect and swelling ratios. The degree of water release could be controlled by the content of DND, light intensity and exposure time. Moreover, the sunlighttriggered water release of the hydrogel beads was reversible and can be repeated multiple times. The composites might "inherit" the super absorbency and tunable biodegradability of SA as well as the high photothermal property of DND, and are very promising for use as water controlled-release carrier materials in agriculture.

\section{Conflicts of interest}

There are no conflicts to declare.

\section{Acknowledgements}

This work was supported by the Innovation Platform for the Development and Construction of Special Project of Key Laboratory of Tibetan Medicine Research of Qinghai Province (No. 2017-ZJ-Y11), Qinghai Provincial Science Foundation (2017-SFA8) and Yulin Municipal Science and Technology Bureau Science Foundation.

\section{References}

1 E. A. Waraich, R. Ahmad, M. Yaseenashraf, S. Ullah and M. Ahmad, Acta Agric. Scand., 2011, 61, 291-304.

2 X. P. Deng, S. Lun, H. Zhang and N. C. Turner, Agric. Water Manag., 2006, 80, 23-40.

3 W. Yang, S. Guo, P. Li, R. Song and J. Yu, J. Sci. Food Agric., 2019, 99, 350-359.

4 S. Jiang, N. Helfricht, G. Papastavrou, A. Greiner and S. Agarwal, Macromol. Rapid Commun., 2018, 39, 1700838.

5 A. Zaharia, A.-L. Radu, S. Iancu, A.-M. Florea, T. Sandu, I. Minca, V. Fruth-Oprisan, M. Teodorescu, A. Sarbu and T.-V. Iordache, RSC Adv., 2018, 8, 17635-17644.

6 P. Davoodi, W. C. Ng, W. C. Yan, M. P. Srinivasan and C. H. Wang, ACS Appl. Mater. Interfaces, 2016, 8, 2278522800 .

7 A. Osman, E. T. Oner and M. S. Eroglu, Carbohydr. Polym., 2017, 165, 61-70.
8 M. Amoli-Diva, R. Sadighi-Bonabi and K. Pourghazi, Mater. Sci. Eng., C, 2017, 76, 242-248.

9 P. H. Dai, G. J. Im, Y. C. Su, C. L. Kang and D. S. Lee, J. Controlled Release, 2009, 137, 20-24.

10 T. H. Cong, S. W. Kang, L. Yi, B. S. Kim and D. S. Lee, Soft Matter, 2011, 7, 8984-8990.

11 J. Lu, Y. Li, D. Hu, X. Chen, Y. Liu, L. Wang and Y. Zhao, BioMed Res. Int., 2015, 2015, 236745.

12 C.-H. Zhu, Y. Lu, J. Peng, J.-F. Chen and S.-H. Yu, Adv. Funct. Mater., 2012, 22, 4017-4022.

13 G. Chang, S. Li, F. Huang, X. Zhang, Y. Shen and A. Xie, J. Mater. Sci. Technol., 2016, 32, 753-762.

14 C. H. Zhu, Y. Lu, J. F. Chen and S. H. Yu, Small, 2014, 10, 2796-2800.

15 X. Zhang, C. L. Pint, M. H. Lee, B. E. Schubert, A. Jamshidi, K. Takei, H. Ko, A. Gillies, R. Bardhan and J. J. Urban, Nano Lett., 2011, 11, 3239-3244.

16 Z. Sobhani, M. A. Behnam, F. Emami, A. Dehghanian and I. Jamhiri, Int. J. Nanomed., 2017, 12, 4509-4517.

17 D. Kim, H. S. Lee and J. Yoon, $R S C A d v ., 2014$, 4, 2537925383.

18 Y. Jang, S. Kim, S. Lee, C. M. Yoon, I. Lee and J. Jang, Chemistry, 2017, 23, 3719-3727.

19 E. Lee, H. Lee, S. I. Yoo and J. Yoon, ACS Appl. Mater. Interfaces, 2014, 6, 16949-16955.

20 R. M. Yang, C. P. Fu, J. Z. Fang, X. D. Xu, X. H. Wei, W. J. Tang, X. Q. Jiang and L. M. Zhang, Int. J. Nanomed., 2017, 12, 197-206.

21 S. Atsushi, M. Takeshi, N. Takuro, N. Yasuro and K. Yoshiki, Langmuir, 2007, 23, 4012-4018.

22 X. Cheng, R. Sun, L. Yin, Z. Chai, H. Shi and M. Gao, Adv. Mater., 2017, 29, 1604894.

23 A. Guo, F. Yang, W. Gang and X. Wang, RSC Adv., 2017, 7, 4815-4824.

24 Z. Xue, J. C. Vinci and L. A. Colon, ACS Appl. Mater. Interfaces, 2016, 8, 4149-4157.

25 M.-C. Kim, D. Lee, S. H. Jeong, S.-Y. Lee and E. Kang, ACS Appl. Mater. Interfaces, 2016, 8, 34317-34326.

26 P. Kai, H. Li, G. Zou, R. Yu, H. Zhao, S. Xi, L. Wang, Y. Song and D. Qiu, J. Power Sources, 2017, 342, 515-520.

27 T. K. Ryu, S. W. Baek, R. H. Kang, K. Y. Jeong, D. R. Jun and S. W. Choi, J. Controlled Release, 2018, 270, 237-245.

28 T.-K. Ryu, S. W. Baek, R. H. Kang and S.-W. Choi, Adv. Funct. Mater., 2016, 26, 6428-6436.

29 S. Osswald, G. Yushin, V. Mochalin, S. O. Kucheyev and Y. Gogotsi, J. Am. Chem. Soc., 2006, 128, 11635-11642.

30 S. T. Kurkin, N. A. Ozerin, S. A. Kechek'yan, A. L. Ozerina, S. E. Obolonkova, A. M. Beshenko and Y. V. Dolmatov, Polym. Sci., Ser. A, 2008, 50, 43-50.

31 E. Tamburri, S. Orlanducci, V. Guglielmotti, G. Reina, M. Rossi and M. L. Terranova, Polymer, 2011, 52, 5001-5008.

32 E. Tamburri, V. Guglielmotti, S. Orlanducci, M. L. Terranova, D. Sordi, D. Passeri, R. Matassa and M. Rossi, Polymer, 2012, 53, 4045-4053.

33 J. Lu, H. Yang, J. Hao, C. Wu, L. Liu, N. Xu, R. J. Linhardt and Z. Zhang, Carbohydr. Polym., 2015, 122, 180-188. 
34 D. Feng, B. Bai, H. Wang and Y. Suo, J. Agric. Food Chem., 2017, 65, 5896-5907.

35 J. Yao, Y. Zhou, X. Chen, F. Ma, P. Li and C. Chen, Food Chem., 2018, 239, 1134-1142.

36 B. Wang, B. Gao, A. R. Zimmerman, Y. Zheng and H. Lyu, J. Environ. Manage., 2018, 209, 105-111.

37 M. V. Dinu, M. M. Perju and E. S. Drăgan, React. Funct. Polym., 2011, 71, 881-890.

38 Z. Delavar and A. Shojaei, Carbohydr. Polym., 2017, 167, 219228.

39 N. F. Attia, J. P. Rao and K. E. Geckeler, J. Nanopart. Res., 2014, 16, 2361.

40 E.-Z. Pina-Salazar, K. Urita, T. Hayashi, R. Futamura, F. Vallejos-Burgos, J. Włoch, P. Kowalczyk, M. Wiśniewski, T. Sakai, I. Moriguchi, A. P. Terzyk, E. Osawa and K. Kaneko, Langmuir, 2017, 33, 11180-11188.

41 O. Shenderova, A. M. Panich, S. Moseenkov, S. C. Hens, V. Kuznetsov and H. M. Vieth, J. Phys. Chem. C, 2011, 115, 19005-19011.

42 S. Su, J. Wei, K. Zhang, J. Qiu and S. Wang, Colloid Polym. Sci., 2015, 293, 1299-1305.

43 R. V. Kulkarni, V. Sreedhar, S. Mutalik, C. M. Setty and B. Sa, Int. J. Biol. Macromol., 2010, 47, 520-527.

44 H. S. Samanta and S. K. Ray, Carbohydr. Polym., 2014, 99, 666-678.

45 T. K. Vidal Urquiza, O. Perales Pérez and M. Gálvez Saldaña, MRS Proceedings, 2011, 1355.

46 V. Pichot, M. Comet, E. Fousson, C. Baras, A. Senger, F. Le Normand and D. Spitzer, Diamond Relat. Mater., 2008, 17, 13-22.

47 M. Chhatbar, R. Meena, K. Prasad and A. K. Siddhanta, Carbohydr. Polym., 2009, 76, 650-656.
48 C. Larosa, M. Salerno, J. S. de Lima, R. Merijs Meri, M. F. da Silva, L. B. de Carvalho and A. Converti, Int. J. Biol. Macromol., 2018, 115, 900-906.

49 L. Bin, W. Bin, L. Minghui, N. Jing, L. Xianglong and Z. Linjie, Adv. Mater., 2012, 24, 1405-1409.

50 M. Ionita, M. A. Pandele and H. Iovu, Carbohydr. Polym., 2013, 94, 339-344.

51 K. Sato, Y. Tominaga, Y. Hotta, H. Shibuya, M. Sugie and T. Saruyama, Adv. Powder Technol., 2018, 29, 972-976.

52 W. Sheng, W. Li, B. Li, C. Li, Y. Xu, X. Guo, F. Zhou and X. Jia, Macromol. Rapid Commun., 2015, 36, 1640-1645.

53 P. Zhao, F. Yu, R. Wang, Y. Ma and Y. Wu, Chemosphere, 2018, 200, 612-620.

54 X. Zhang, X. Lin, Y. He and X. Luo, Int. J. Biol. Macromol., 2019, 136, 445-459.

55 G. Zain, A. A. Nada, M. A. El-Sheikh, F. A. Attaby and A. I. Waly, Int. J. Biol. Macromol., 2018, 115, 61-68.

56 D. Cheng, Y. Liu, G. Yang and A. Zhang, J. Agric. Food Chem., 2018, 66, 5762-5769.

57 A. M. Abdallah, International Soil and Water Conservation Research, 2019, 7, 275-285.

58 A. K. Bhardwaj, I. Shainberg, D. Goldstein, D. N. Warrington and G. J. Levy, Soil Sci. Soc. Am. J., 2007, 71, 406-412.

59 Y. Pan, Micro Nano Lett., 2010, 5, 237-240.

60 T. Singh and R. Singhal, J. Appl. Polym. Sci., 2013, 129, 31263139.

61 M. Evren, l. Acar, K. Güçlü and G. Güçlü, Can. J. Chem. Eng., 2013, 92, 52-59.

62 A. K. Bajpai and A. Giri, Carbohydr. Polym., 2003, 53, 271279.

63 H. El-Hamshary, Eur. Polym. J., 2007, 43, 4830-4838. 